Document downloaded from:

http://hdl.handle.net/10251/145992

This paper must be cited as:

Ibanez-Del Valle, V.; Silva, J.; Castello-Domenech, A.; Martinez-Martinez, M.; Verdejo, Y.; Sanantonio-Camps, L.; Cauli, O. (05-2). Subjective and objective sleep quality in elderly individuals: The role of psychogeriatric evaluation. Archives of Gerontology and Geriatrics. 76:221-226. https://doi.org/10.1016/j.archger.2018.03.010

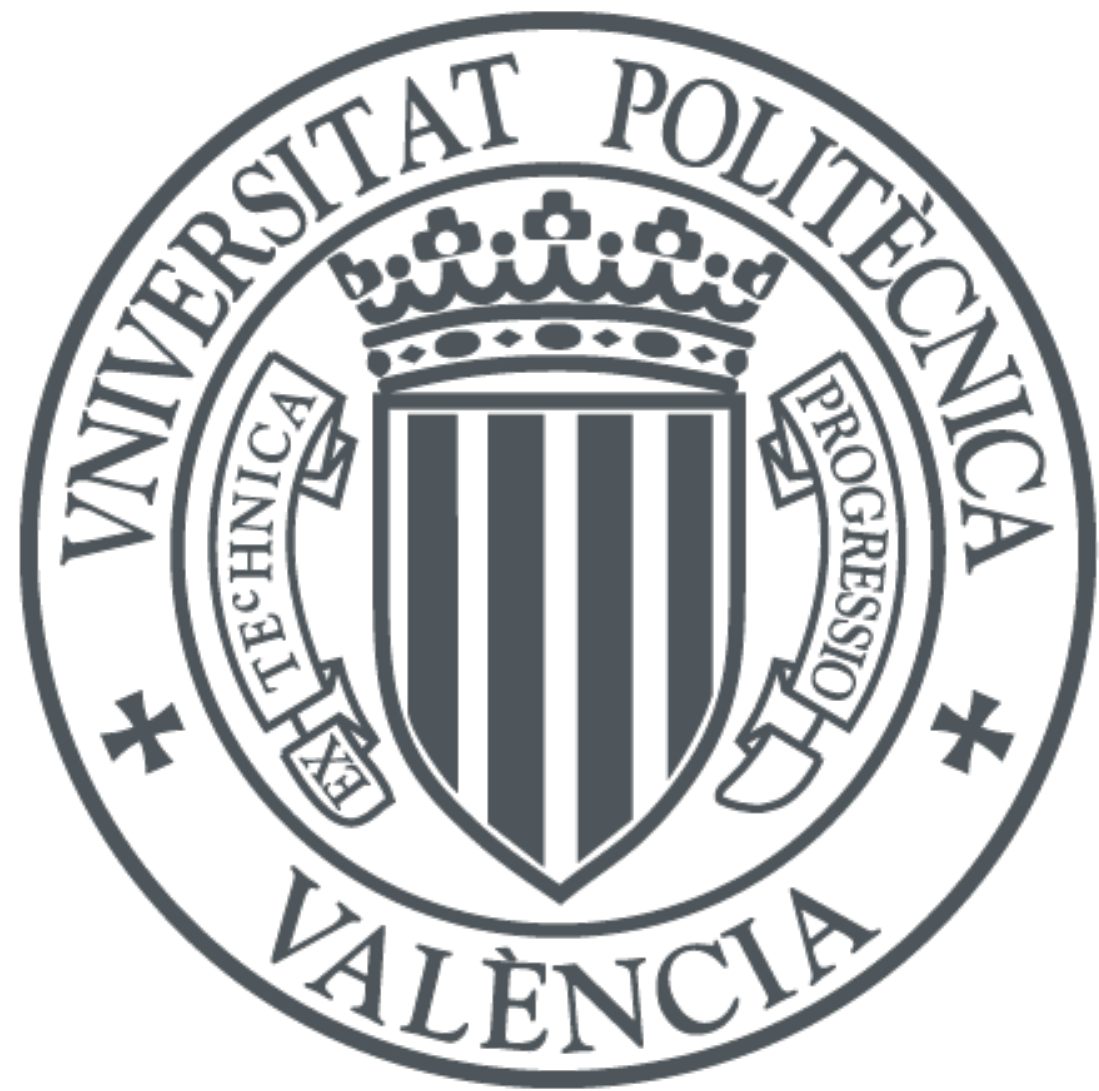

The final publication is available at

https://doi.org/10.1016/j.archger.2018.03.010

Copyright Elsevier

Additional Information 


\title{
Subjective and objective sleep quality in non-demented elderly individuals: role of psychogeriatric
} evaluation.

\begin{abstract}
Aging affects sleep, and sleep problems are common in older individuals but the relationship between objective or subjective tool to analyse sleep and psycho-geriatric variables have not been in institutionalised older individuals. This work analyse sleep quality by means of objective tool (with actigraphy) and subjective perception of sleep by validates scales (measured with the Athens and Oviedo sleep questionnaires) in octogenarian non-demented elderly individuals. All patients wore an actigraph device for one week and then completed the Athens and Oviedo clinical sleep-evaluation questionnaires. Morning cortisol levels in blood plasma and saliva samples were also measured in order to assess any associations with the reported sleep patterns. Age, gender, and psycho-geriatric evaluations, including Barthel, Tinetti, and Mini-Mental scale measurements, were analysed as variables with the potential to confound the strength of such associations. There was a significant and inverse correlation between the number of awakenings during the night assessed by actigraphy or the time spent awake during night and the total Oviedo questionnaire score, but he other parameters were not. The concentration of blood cortisol appears of marker of insomnia, being related to sleep times of less than four hours and represent a potential marker for sleep interventions.
\end{abstract}

Keywords: sleep, actigraphy, Athens questionnaire, Oviedo questionnaire, cortisol, elderly people 


\section{Introduction}

Sleep disorders are common in elderly populations (Rodriguez et al., 2015) and aging can influence sleep patterns (Foley et al., 1995; Mellinger et al., 1985). When sleep alterations appear, they can significantly reduce quality of life and promote adverse outcomes in other pathologies (Sariarslan et al., 2015). Although some older adults complain of poor night-time sleep and subsequent impairments in daytime functioning, others assume that their difficulties are part of the normal aging process and so do not complain about their sleep problems, even when the quality of their sleep is impaired. The mechanisms behind sleep misperception are still poorly understood and thus, the comparison of objective and subjective sleep quality has more recently become a research target in relation to different diseases, e.g. (Hasselberg et al., 2013; Chen et al., 2015; Klumpp et al., 2017), such as depression or diseases characterized by cognitive impairment, where both sleep quality and the perception of sleep are of paramount importance.

Because questionnaires are the most commonly used instruments for detecting sleep problems, it is crucial that subjective descriptions of sleep quality be correlated with its objective measurement with tools such as actigraph devices in order to implement interventions to improve sleep duration, maintenance, or sleep onset latency. Recent studies (Landry et al., 2015; Chen et al., 2015; Klumpp et al., 2017) used actigraphy as an objective measurement of sleep quality because technological advances in actigraph instruments have improved their precision in measuring sleep in patients' normal environments (e.g., at home) for long periods of time. In contrast, polysomnography can only be administered for very small periods of time, and the sleep is assessed outside patients' normal contexts (e.g., in a hospital). In this latter technique, patients are hooked up to machines via up to 20 electrodes placed on their body and head, in a laboratory setting with cameras rather than in their own bed. Hence, in this context, it is understandable that subjects might not sleep normally. Moreover, recent validation studies focusing on actigraphy have justified their use as an objective measurement of sleep, e.g. (Meadows et al., 2010; Meltzer et al., 2016).

Many sleep-related disorders are underdiagnosed in older individuals, and others respond poorly to pharmacological treatments. Moreover, psychotropic drugs can induce a wider variety of side effects in older individuals compared to younger populations and so it is important to evaluate their utility in the management of sleep problems specifically in older individuals. Therefore, the main aim of this present study was to perform a focused evaluation of sleep quality, correlating objective measurement (actigraphy) and the corresponding subjective evaluation tool (Oviedo and Athens questionnaire) results, in relation to geriatric evaluation scales. Because changes in cortisol have been linked to sleep alterations in older 
individuals (Castello-Domenech et al., 2016; Vgontzas et al., 1999; Kushida, 2004; Bush \& Hudson, 2010) we also evaluated the relationship between the morning concentration of cortisol in blood and saliva in these patients and their sleep quality. Thus, we investigated the following three objectives:

(1) Evaluate sleep quality in older institutionalized individuals by actigraphy and validated sleep-related questionnaires.

(2) Evaluate the relationship between sleep quality and psycho-geriatric evaluation.

(3) Evaluate the association between morning cortisol concentrations and sleep quality and efficiency.

\section{Methods}

\subsection{Design and study population}

This was a cross-sectional design research study conducted between 2014 and 2016 with institutionalized elderly individuals living in different nursing homes located in Valencia and its province (GeroResidencias La Saleta, Valencia). The inclusion criteria were: institutionalization for at least 6 months, ability to understand the sleep-quality questionnaire questions, and age of 60 years or older. The exclusion criteria were severe cognitive impairment or inability to speak, poorly-controlled psychiatric disease (e.g., schizophrenia, bipolar disorder, etc.), blindness, or the presence of acute infections, known cancer, or active corticoid treatment. The research complied with the requirements of the Declaration of Helsinki and the entire study protocol was approved by the local ethics committee at the University of Valencia (approval reference number: H1384175284261). All the participants signed a written informed consent form. A total of 62 (out 93 fulfilling inclusion criteria) patients from six different residences for the elderly signed the consent and thus, participated in the study. Sociodemographic and pharmacotherapeutic treatment data were collected, and the Charlson comorbidity index (Charlson et al., 1987) was calculated in order to quantify the burden of pluripathologies.

\subsection{Evaluation of subjective quality of sleep}

Sleep quality was measured using two validated tools: the Athens insomnia scale (Soldatos et al., 2000) and the Oviedo questionnaire (Bobes et al., 1998). Data were collected by trained personnel via face-to-face evaluations. The Athens insomnia scale (AIS) is a self-administered psychometric instrument designed to measure sleep difficulty based on the diagnostic criteria of the 10th revision of the international statistical classification of diseases and related health problems (ICD-10) and has been validated in a Spanish 
population (Jiménez Genchi \& Nenclares Portocarrero, 2005). It consists of eight items scored from 0 to 3 : the higher the score, the greater the difficulty in sleeping. The first five factors are related to night-time sleep and the last three factors are related to daytime dysfunction. A cut-off score of $\geq 6$ on the AIS is used to establish the diagnosis of insomnia (Soldatos et al., 2002).

The Oviedo sleep quality questionnaire (SQQ) provides diagnostic help for insomnia- and hypersomniatype disorders according to the 4th edition of the diagnostic and statistical manual of mental disorders (DSM-IV) and ICD-10 criteria. The SQQ comprises 15 items; 13 are grouped into three subscales of: subjective sleep satisfaction (one item), insomnia (nine items), and hypersomnia (three items); the remaining two items give information about other organic alterations that can induce sleep disorders such as snoring with awakenings, nightmares, and restless legs syndrome, etc. and the use of non-prescription drugs or herbal infusions to induce sleep. The insomnia subscale explores different dimensions such as sleep latency, duration, efficiency, and diurnal dysfunction due to altered sleep.

All the items are answered using a Likert-type scale from 1 to 5, except the subjective sleep satisfaction item (measured on a scale of 1 to 7 ). The questionnaire also provides a score of the severity of the insomnia (range: 9-45).

\subsection{Actigraphy}

Participants were recruited at their residences. A specialist nurse programmed the actigraph units, informed the patients about how to wear them, and downloaded the collected data. The actigraph devices were configured with 1-min epochs (i.e., a sampling length of one minute); the raw data collected from the actigraph is provided from its internal accelerometer. The accelerometer only provides proper acceleration the XYZ dimensions and so software is required to transform and analyze these data; in our case we used ActiLife ${ }^{\circledR}$ software. ${ }^{1}$ In particular, sleep was detected using the Cole-Kripke algorithm (CKA) available from ActiGraph ${ }^{\circledR}$ (Cole et al., 1992) because it is the most appropriate algorithm for older individuals (Actigraphcorp.com, 2015). For instance, the filled areas in the charts in Figure 1 representing sleep periods were determined with the CKA.

\footnotetext{
${ }^{1}$ http://actigraphcorp.com/products-showcase/software/actilife/
} 
ActiLife $^{\circledR}$ produces processed data that contains every single sleep period detected by the CKA for every patient. Of course, there may be several sleep periods for one patient within the same day. From this information, ActiLife ${ }^{\circledR}$ can generate different reports but, unfortunately, it does not allow us to further transform or manipulate the data displayed. We wanted to produce more sophisticated reports and so we developed a complementary software system to extract additional derived and aggregated information from the raw ActiLife ${ }^{\circledR}$ data files. This analyzer produces additional information including: average time spent asleep per day (including and excluding naps); average time spent in bed per day (including and excluding naps); total time spent in bed in a wakeful state per day. The main information collected for each patient is summarized in Table 1. It includes the actigraphy information, the Oviedo and Athens questionnaire and blood and saliva test results, and the medical report on diseases, medications, etc. for each patient. Information on demographics, current and past diseases, medical conditions, prescription of drugs (including sleep medications), diagnosis of psychiatric and sleep disorders, as well as any daily activities that might affect sleep/wake behaviors were also collected.

\subsection{Measurement of cortisol}

Saliva samples were obtained in the morning between 9:00-11:00 a.m. in non-fasting conditions. Briefly, the procedure consisted of deproteinising $100 \mu \mathrm{L}$ of saliva by adding $1000 \mu \mathrm{L}$ dichloromethane; samples were gently agitated and centrifuged at $3500 \mathrm{rpm}$ for 15 minutes at room temperature. The supernatant was collected, evaporated under nitrogen, and the residue was re-suspended in $100 \mu \mathrm{L}$ of ethyl acetate. Cortisol concentrations were assessed by high-performance liquid chromatography coupled to mass spectroscopy. The concentration of cortisol $(\mathrm{mg} / \mathrm{dL})$ in the samples was extrapolated from a standard cortisol solution curve that covered the range of values found in the samples. The expert consensus on measuring waking cortisol states that at least three post-awakening cortisol samples are required from the same person on at least on two different days to determine the peak level (Stalder et al., 2016) and so, all the values in this study were obtained from the mean of three measurements made over a period of 11-15 days. All the reagents were purchased from Sigma-Aldrich.

\subsection{Statistical analysis}

The results are presented as the mean \pm standard deviation, and the Shapiro-Wilk test was used to check for a normal data distribution. All the quantitative variables in the study had a non-normal distribution and so we used non-parametric statistical analysis tools: differences between the groups were analyzed using the 
Kruskal-Wallis test and bivariate correlations between variables were evaluated using the Spearman correlation test as well as multivariable-adjusted statistical analyses. $P$ values $<0.05$ were considered to be statistically significant. All the statistical analyses were performed using SPSS software (version 20.0; SPSS, Inc., Chicago, IL).

\section{Results}

\subsection{Clinical features of the sample}

We included an extensive demographic and medical information for each participant in order to analyse any possible association with the sleep variables. Regarding marital status, the sample was $19.51 \%$ single, $14.63 \%$ married, $56.10 \%$ widowed, $2.44 \%$ divorced, and $7.32 \%$ with an 'other' status. Regarding pharmacotherapy with drugs which act at the level of central nervous system, $29.27 \%$ of the patients took antipsychotics, $41.46 \%$ took antidepressants, $48.78 \%$ took hypnotics, $43.9 \%$ took anxiolytics, $19.51 \%$ took antiepileptics, and $80.49 \%$ took opioids/analgesics. During the time the patients wore the actigraph units, each patient had been prescribed an average of 11.20 drugs, of which 3.28 acted on the central nervous system. The psycho-geriatric information about the sample, including the Barthel, Tinetti, and Mini-Mental scale results, is shown in Table 2.

\subsection{Evaluation of sleep by the Athens Scale and Oviedo questionnaire}

The results obtained using the Athens and Oviedo scales are summarized in Table 3. The mean Athens scale score was $4.00 \pm 3.99$ from a minimum score of 0 (no sleep disorder) and a maximum score of 16 . According to the Athens Scale criteria, the presence of a sleep disorder is considered when the score is 6 or more (Soldatos et al., 2000) thus, according to this criterion, $20 \%$ of our patients had a sleep disorder.

Regarding the Oviedo scale, the total mean score was $29.48 \pm 6.85$; the average sleep satisfaction subscale (Oviedo subscale 1) score was $3.88 \pm 2.11$, where the patient's subjective satisfaction with sleep ranged from 1 to 7 , with higher numbers signifying higher satisfaction. On the other subscales, higher numbers signified more problematic sleep. The average insomnia subscale (Oviedo subscale 2) score was $20.45 \pm 6.89$ and the average hypersomnia (Oviedo subscale 3) score was $5.15 \pm 3.29$; the average Oviedo subscale 4 ("sleep aid use or presence of adverse events during sleep") score was $8.05 \pm 3.43$. As expected, there was a significant correlation between the Athens scale and the Oviedo subscale sleep satisfaction (rho $=-0.51 p<0.01$, Spearman correlation test) and insomnia (rho $=0.62 p<0.01$, Spearman correlation test) scores, as well as the overall Oviedo score (rho $=0.57 p<0.01$, Spearman correlation test). The Athens 
scale could not be correlated to the Oviedo hypersomnia, sleep aid use subscales, or presence of adverse events during sleep because the former does not evaluate these items. However, age and the Charlson comorbidity index score did significantly correlate (rho $=0.32, P<0.05$, Spearman correlation test), although neither of them significantly correlated with the Athens scale or any of the Oviedo subscales; no significant correlation was found between age and sleep problems.

\subsection{Evaluation of sleep by actigraphy}

Each participant was asked to wear a wGT3X-BT ${ }^{\circledR}$ actigraph device for one week. After our initial data analysis, we discarded 11 patients because the data collected for them was anomalous-for example, they stopped wearing the actigraph unit for long periods of time which invalidated their data. Hence, the final sample was $\mathrm{N}=41$ patients [ $72 \%$ females, aged 63-97 years].

The actigraph devices collected valuable objective data about the sleep patterns of our subjects (see Table 3). In particular, they indicated that among our patients the sleep efficiency (time asleep/time in bed) was $95.31 \% \pm 2.57 \%$, the total sleep time was $321.92 \pm 105.84$ minutes, the average number of awakenings during sleep was $5.47 \pm 3.32$, and the mean awakening length was $2.59 \pm 1.01$ minutes.

\subsection{Correlation between actigraphy and subjective evaluation of sleep}

In order to analyze the relationship between the objective sleep data recorded by actigraphy and the subjective quality of sleep the subjects reported via the Athens and Oviedo questionnaires, we analyzed the correlation between these variables. There was a significant and inverse correlation between the number of awakenings during the night assessed by actigraphy (Figure 2a) or the time spent awake during night (Figure $2 b$ ) and the total Oviedo questionnaire score ( $r h o=-0.32, p<0.05$, and rho $=-0.37, p<0.05$, respectively). No significant effect was observed for the other actigraphy measurements and the other Oviedo questionnaire subscales or with the Athens scale score. However, there was a correlation between diagnosis of insomnia by the Athens questionnaire (AIS $\geq 6$ (Soldatos et al., 2002)) and the number of awakenings during night and the time spent awake during the night (rho $=-0.34, p<0.05$, and rho $=-0.38, p<0.05$, respectively).

There was a significant and inverse correlation between age and total sleep time recorded by actigraphy (rho $=-0.33 P<0.05$, Spearman correlation test; Figure $3 \mathrm{a}$ ) and with the time spent in bed 
during sleeping (rho $=-0.32 P<0.05$, Spearman correlation test; Figure $3 b$ ). The same correlation was observed in (Unruh et al., 2008).

\subsection{Correlation between actigraphy and cortisol concentration, Charlson index, and administered drugs}

We measured both the saliva and blood plasma cortisol concentrations $(\mathrm{mg} / \mathrm{dL})$ collected during the morning in order to study their relationship with the sleep in our targeted population, controlling for the collection time as a confounding variable. No significant correlation was observed between the saliva cortisol concentration and any of the sleep measurements, however blood plasma cortisol concentration significantly correlated with sleep time; in particular, patients that slept a mean of 4 hours or less presented high blood plasma cortisol concentrations ( $r$ o $=0.56, p<0.01$, Spearman correlation test). No significant effect was observed between the Charlson index score or the number of drugs administered daily.

\section{Discussion}

The International Classification of Sleep Disorders coined the term sleep state misperception to refer to people who wrongly perceive their sleep as wakefulness, or who severely overestimate their sleep time. This phenomenon has been studied for people aged 19-84 with insomnia and sleep apnea (Soldatos et al., 2000), where the authors reported that the subjective perception of sleep does not necessarily match the objective measures for this population. In this work, we targeted another population for which sleep disorders are common: institutionalized elderly populations (aged over 62 years). Studies have shown that this population frequently experiences prolonged sleep latency, increased sleep fragmentation, and episodes of waking after sleep onset, more disturbed circadian rhythms, and night-day reversal (Meadows et al., 2010).

Our study sample is particularly interesting because it is novel with respect to previous studies using actigraphy which targeted other populations and age groups such as children (aged 5-12) (Meltzer et al., 2016), adults (aged 23-47) (Marino et al., 2013; Bianchi et al., 2013), patients with schizophrenia or bipolar disorder (Baandrup \& Jennum, 2015), or elderly women (mean age 81.9 years) (Blackwell, 2008), among others (see (Martin \& Hakim, 2011; Marino et al., 2013) for a comparison of these studies). This was the first study to include quantitative data regarding objective measures of sleep quality (sleep efficiency, total sleep time, number of awakenings, etc.) and cortisol concentrations, among the other variables studied (see Table 1), for institutionalized individuals. The most similar work to ours is (Meadows et al., 2010), which 
targets the same population but studies other variables (inter-day stability, intra-day variability, relative amplitude of the activity rhythm, and mean level of activity during the 24-hour day). Thus, neither the sample population nor the Athens and Oviedo questionnaires have been used for this purpose in previous studies.

Our study reveals that the mean sleep time in this population is less than five and a half hours, and the average sleep efficiency is over $95 \%$. This is slightly under the average (Ohayon et al., 2004), and far below expert recommendations (e.g., in the American National Sleep Foundation) of 7-8 hours a day for people older than 65 years. Regarding the subjective measurements, our study revealed a correlation between the subjective and objective perception of sleep in this population. In particular, the number of awakenings during the night and their total length correlates with both the SQQ scale score and the AIS diagnosis of insomnia. Therefore, we can conclude that both questionnaires are valid for the diagnosis of insomnia in this targeted population. It is, however, important to note that none of the Oviedo subscales were correlated with any sleep measurements, even the insomnia subscale. Similarly (Landry et al., 2015) incorporated a consensus sleep diary as the subjective measure of sleep and noted that these measurements survey different aspects of sleep quality compared to the objective measures. They concluded that an older adult's perception of their sleep quality is quite different from the objective reality.

Interestingly, (Chen et al., 2015) and (Klumpp et al., 2017) are similar studies which targeted different populations; while (Chen et al., 2015) looked at individuals with osteoarthritis, (Klumpp et al., 2017) targeted patients with social or generalized anxiety disorder, and/or major depressive disorder. These studies suggest that when considering a group of patients with specific diseases, the strength of correlation, if any, can vary considerably. In our sample, no correlation was found between the subjective perception of sleep and any of the psycho-geriatric information (Barthel, Tinetti, Mini-Mental test results) suggesting that sleep is not directly related to other alterations found in older individuals.

It is also worth noting the inverse correlation we found between age and the overall sleep time, which has been also reported in previous studies (Ohayon et al., 2004); this confirms that the need for and duration of sleep progressively decreases over time and with aging. The interaction of the hypothalamic-pituitaryadrenal (HPA) axis with sleep is well-known and has been previously studied, e.g. (Leproult et al., 1997; Backhaus et al., 2004; Bush \& Hudson, 2010). In particular, HPA axis hyperactivity can lead to shortened sleep time, among other sleep disorders (Bush \& Hudson, 2010). This aligns with the correlation found in our study that relates high blood plasma cortisol concentration levels with shortened sleep time ( $<4$ hours). 
However, it is of note that sleep problems did not correlate with saliva cortisol concentrations. This contrasts with other studies such as (Backhaus et al., 2004), although these were performed in a younger population (aged 32-62 years). Hence, our findings strongly suggest that blood plasma rather than saliva cortisol concentrations should be used as a marker for detecting sleep problems for this older population, although longitudinal studies will still be required to fully understand any causal relationships in this regard.

While cortisol is essential for normal body function, among other consequences, sustained high levels of cortisol destroys healthy muscle and bone, interferes with healthy endocrine function, impairs digestion, slows down healing, normal cell regeneration, metabolism, and mental function, and weakens the immune system. Therefore, according to our findings, cortisol should be regularly measured in institutionalized individuals who sleep less than 4 hours. Detection of high levels of cortisol or shortened sleep times ( $<4$ hours) should trigger protocols to reduce anxiety and promote relaxation and sleep in this population. These protocols should include interventions to promote sleep hygiene and enhancement and management to provide a relaxing environment that favors comfort and, thus, sleep.

Among the measures which are fundamental for sleep hygiene in this population, of special importance are: (1) maintaining a regular sleep/wake schedule, (2) avoiding afternoon or evening napping, (3) avoiding forced sleep, (4) avoiding alcohol and caffeine in the evening, (5) avoiding stressful activities at bedtime, (6) minimizing annoying noise, light, or temperature extremes, (7) practicing relaxation exercises before going to bed, and (8) avoiding large meals. In addition, non-pharmacological interventions have also been shown to be effective at producing long-term changes in the sleep habits of individuals with chronic insomnia (Beltran et al., 2014; Bikowski et al., 2001) and different authors propose nonpharmacological measures as the first-line treatment for insomnia (NANDA, 2014; Dochterman \& Bulechek, 2007; Kalyanakrishan \& Dewey, 2007). Thus, such measures should also be considered as a complement or alternative for elderly patients.

Apart from saliva cortisol concentrations, other variables such as the comorbidity index (Charlson index) or the number of drugs administered daily did not correlate with our actigraphy measurements suggesting that the disease burden of these patients may not be responsible for their sleep alterations or the cause of their sleep-related problems. Our results suggest that a thorough analysis of sleep-related impairment in older institutionalized individuals necessarily requires some clinical variables, such blood plasma cortisol concentrations (for people with very short nighttime sleep durations), be taken into account, and that age, gender, gait impairment (expressed by the Tinetti score in our study) should be considered in 
order to control for variables that may be related to sleep quality. This complex analysis is crucial to be able to adequately tailor therapeutic strategies so that sleep quality can be improved in older institutionalized individuals in which poor sleep quality is a prevalent disorder.

\section{Disclosure Statement:}

Authors declare no conflict of interest.

\section{References}

Rodriguez, J. C., Dzierzewski, J. M., \& Alessi, C. A. (2015). Sleep Problems in the Elderly. Medical Clinics of North America, 99(2): 431-439.

Foley, D. J., Monjan, A. A., Brown, S. L., Simonsick, E. M., Wallace, R. B., \& Blazer, D. G. (1995). Sleep complaints among elderly persons: an epidemiologic study of three communities. Sleep, 18 (6):425-32.

Mellinger, G. D., Balter, M. B., \& Uhlenhuth, E. H. (1985). Insomnia and its treatment. Prevalence and correlates. Archives of General Psychiatry, 42(3):225-32.

Sariarslan, H. A., Gulhan, Y. B., Unalan, D., Basturk, M., \& Delibas, S. (2015). The relationship of sleep problems to life quality and depression. Neurosciences (Riyadh), 20(3):236-242.

Hasselberg, M. J., Porsteinsson, A. P., Boyle, L., \& Parker, K. P. (2013). Subjective and Objective Measures of Sleep Quality in Advanced Cancer: A Possible Clinical Marker for Depression. Journal of Sleep Disorders \& Therapy; 2:135.

Chen, C. J., McHugh, G., Campbell, M., \& Luker, K. (2015). Subjective and Objective Sleep Quality in Individuals with Osteoarthritis in Taiwan. Musculoskeletal care, 13(3):148-159.

Klumpp, H., Roberts, J., Kapella, M. C., Kennedy, A. E., Kumar, A., \& Phan, K. L. (2017). Subjective and objective sleep quality modulate emotion regulatory brain function in anxiety and depression. Depression and Anxiety; 34(7):651-660.

Landry, G. J., Best, J. R., \& Liu-Ambrose, T. (2015). Measuring sleep quality in older adults: a comparison using subjective and objective methods. Frontiers in Aging Neuroscience, 7:166. 
Meadows, R., Luff, R., Eyers, I., Venn, S., Cope, E., \& Arber, S. (2010). An actigraphic study comparing community dwelling poor sleepers with non-demented care home residents. Chronobiology International, 27(4):842-54.

Meltzer, L. J., Wong, P., Biggs, S. N., Traylor, J., Kim, J. Y., Bhattacharjee, R., Narang, I., \& Marcus, C. L. (2016). Validation of actigraphy in middle childhood. Sleep, 39(6):1219-24.

Castello-Domenech, A. B., Ibáñez Del Valle, V., Fernandez-Garrido, J., Martinez-Martinez, M., \& Cauli, O. (2016). Sleep alterations in non-demented older individuals: The role of cortisol. Endocrine, Metabolic \& Immune Disorders - Drug Targets, 16(3):174-180.

Vgontzas, A. N., Mastorakos, G., Bixler, E. O., Kales, A., Gold, P. W., \& Chrousos, G. P. (1999). Sleep deprivation effects on the activity of the hypothalamic-pituitary-adrenal and growth axes: potential clinical implications. Clinical Endocrinology, 51(2):205-15.

Kushida, C.A. (2004). Sleep deprivation: basic science, physiology and behavior. Lung Biology in Health and Disease, volume 192.

Bush, B., \& Hudson, T. (2010). The role of cortisol in sleep. Natural Medicine Journal, 2(6).

Charlson, M. E., Pompei, P., \& Ales, K. L., et al., (1987). A new method of classifying prognostic comorbidity in longitudinal studies: development and validation, Journal of Chronic Diseases, 40(5):373383.

Soldatos, C. R., Dikeos, D. G., \& Paparrigopoulos, T. J. (2000). Athens insomnia scale: validation of an instrument based on ICD-10 criteria. Journal of Psychosomatic Research, 48:555-560.

Bobes, J., González, M. P., Vallejo, J., Sáiz, J., Gibert, J., Ayuso, J. L., \& Rico, F. (1998). Oviedo Sleep Questionnaire (OSQ): A new semistructured Interview for sleep disorders. European Neuropsychopharmacology, 8(2):S162.

Jiménez Genchi, A., \& Nenclares Portocarrero, A. (2005). Estudio de validación de la traducción al español de la escala Atenas de insomnio. Salud Mental, 28 (5).

Soldatos, C. R., Dikeos, D. G., \& Paparrigopoulos, T. J. (2002). The diagnostic validity of the Athens insomnia scale. Journal of Psychosomatic Research. 55: 263-7. 
Cole, R. J., Kripke, D. F., Gruen, W., Mullaney, D. J., \& Gillin, J. C. (1992). Technical note: automatic sleep/wake identification from wrist actigraphy. Sleep, 15: 461-469.

Actigraphcorp.com (2015). ActiGraph White Paper: Actigraphy Sleep Scoring Algorithms. Available online: http://actigraphcorp.com/wp-content/uploads/2015/06/Sleep-Scoring-White-Paper.pdf.

Stalder, T., Kirschbaum, C., Kudielka, B. M., Adam, E. K., Pruessner, J. C., Wüst, S., Dockray, S., Smyth, N., Evans, P., Hellhammer, D. H., Miller, R., Wetherell, M. A., Lupien, S. J., \& Clow A. (2016). Assessment of the cortisol awakening response: Expert consensus guidelines. Psychoneuroendocrinology, 63:414-32.

Unruh, M. L., Redline, S., An, M. W., Buysse, D. J., Nieto, F. J., Yeh, J. L., \& Newman, A. B. (2008). Subjective and objective sleep quality and aging in the sleep heart health study. Journal of the American Geriatrics Society; 56(7):1218-1227.

Marino, M., Li, Y., Rueschman, M. N., Winkelman, J. W., Ellenbogen, J. M., Solet, J. M., Dulin, H., Berkman, L. F., \& Buxton, O. M. (2013). Measuring sleep: accuracy, sensitivity, and specificity of wrist actigraphy compared to polysomnography. Sleep, 36(11):1747-55.

Bianchi, M. T., Williams, K. L., Mckinney, S., \& Ellenbogen, J. M. (2013). The subjective-objective mismatch in sleep perception among those with insomnia and sleep apnea. Journal of Sleep Research, 22: 557-568.

Baandrup, L., \& Jennum, P. J. (2015). A validation of wrist actigraphy against polysomnography in patients with schizophrenia or bipolar disorder. Journal of Neuropsychiatric Disease and Treatment, 11:22712277.

Blackwell, T., Redline, S., Ancoli-Israel, S., Schneider, J. L., Surovec, S., Johnson, N. L., Cauley, J. A., \& Stone, K. L. (2008). Comparison of sleep parameters from actigraphy and polysomnography in older women: the SOF study. Sleep, 31(2):283-91.

Martin, J. L., \& Hakim, A. D. (2011). Wrist Actigraphy. Chest, 139(6):1514-1527.

Ohayon, M. M., Carskadon, M. A., Guilleminault, C., \& Vitiello, M. V. (2004). Meta-analysis of quantitative sleep parameters from childhood to old age in healthy individuals: developing normative sleep values across the human lifespan. Sleep, 27(7):1255-73. 
Leproult, R., Copinschi, G., Buxton, O., \& Van Cauter, E. (1997). Sleep loss results in an elevation of cortisol levels the next evening. Sleep, 20(10):865-870.

Backhaus, J., Junghanns, K., \& Hohagen, F. (2004). Sleep disturbances are correlated with decreased morning awakening salivary cortisol. Psychoneuroendocrinology, 29:1184-1191.

Bush, B., \& Hudson, T. (2010). The role of cortisol in sleep. Natural Medicine Journal, 2:6.

Beltran, M., Fobelo, M. J., Gomez, M. J., \& Bejarano, D. (2014). Manejo terapéutico del paciente anciano. Hospital Universitario de Valme, SEFH.

Bikowski, R., Ripsin, C., \& Viki, L. (2001). Physician-patient congruence regarding medication regimens. Journal of the American Geriatrics Society, 49(10):1353-1357.

NANDA (2014). North American Nursing Diagnosis Association. Nursing Diagnoses 2015-17: Definitions and Classification, ISBN: 978-1-118-91492-2.

Dochterman, J. M., \& Bulechek, G. M. (2007). Nursing Interventions Classification (NIC) (Fifth Edition). ISBN: 9780323053402.

Kalyanakrishan, R., \& Dewey, C. (2007). Treatment options for insomnia. American Family Physician, 76:517-26. 
Table 1: Demographic information collected for each patient and variables used in the analyses.

\begin{tabular}{|l|l|l|}
\hline Demographics & \multicolumn{1}{|c|}{ Actigraphy } & \multicolumn{1}{c|}{ Drugs } \\
\hline Gender & In bed time & Antipsychotics \\
Age & Out bed time & Antidepressants \\
Marital status & Efficiency & Hypnotics \\
Internship & Total time in bed & Anxiolytics \\
& Total sleep time & Antiepileptics \\
& Waking after sleep onset \\
& Number of awakenings \\
& Avg. awakening time & Analgesics \\
& \multicolumn{1}{|c|}{ Morbidities } & Saliva/Blood Test \\
\hline Sleep Scales & Charlson index & Cortisol \\
\hline $\begin{array}{l}\text { Oviedo } \\
\text { Athens }\end{array}$ & & \\
\hline
\end{tabular}


Table 2: Psycho-geriatric information for the sample

\begin{tabular}{|l|l|}
\hline Gender & $72 \% \mathrm{~F}, 28 \% \mathrm{M}$ \\
\hline Age & Mean \\
\hline Barthel (performance in activities of daily living) & $82.76 \pm 8.70$ \\
\hline Tinetti (risk of falls) & $22.29 \pm 11.90(\max 28)$ \\
\hline Mini-Mental (dementia diagnosis) & $26.25 \pm 7.76(\max 35)$ \\
\hline Charlson Index (ten-year mortality prediction) & $5.98 \pm 2.76(\max 37)$ \\
\hline
\end{tabular}


Table 3: Sleep variable information for the sample

\begin{tabular}{|l|l|}
\hline \multicolumn{1}{|c|}{ Variable } & \multicolumn{1}{c|}{ Mean } \\
\hline Athens Scale & $4.00 \pm 3.99$ \\
\hline Oviedo Subscale 1 (sleep satisfaction) & $3.88 \pm 2.11$ \\
\hline Oviedo Subscale 2 (insomnia) & $20.45 \pm 6.89$ \\
\hline Oviedo Subscale 3 (hypersomnia) & $5.15 \pm 3.29$ \\
\hline Oviedo Subscale 4 (organic alterations) & $8.05 \pm 3.43$ \\
\hline Oviedo Scale (Total Score) & $29.48 \pm 6.85$ \\
\hline Sleep Efficiency & $95.14 \% \pm 2.57 \%$ \\
\hline Total Sleep Time (min) & $321.92 \pm 105.84$ \\
\hline Number of Awakenings & $5.47 \pm 3.32$ \\
\hline Average Awakening Length (min) & $2.59 \pm 1.01$ \\
\hline
\end{tabular}


Figure 1: Example of the activity recorded over one day with an wGT3X-BT ${ }^{\circledR}$ actigraph unit Figure 2: Correlation between the objective and subjective perception of sleep

Figure 3: Correlation between age and sleep habits 
